\title{
MS14-04 | Modularity in Minerals: The Example Of Biopyriboles-Palysepioles
}

Nespolo, Massimo (Université de Lorraine, Vandoeuvre-lès-Nancy, FRA); Umayahara, Akihiro (Université de Lorraine and Radboud University Nijmegen, Vandoeuvre-lès-Nancy and Nijmegen, FRA)

Modularity expresses the way in which the same building blocks, larger than the coordination polyhedron, occur in various crystal structures, which can therefore be gathered in series of structurally related compounds. Two wellknown examples are those of biopyriboles and palysepioles, which have been described as two polysomatic series. A new unifying scheme describes all the minerals in these two series as built by TOT rods of variable width extracted from the structure of phyllosilicates. The close structural similarity between any two members of these two series can be shown by expressing the fractional atomic coordinates in a common reference. Accordingly, the two polysomatic series can actually be interpreted as a single series whose prototype is a phyllosilicate

The operations mapping pairs of modules in each of the member of this series are partial operations; the symmetry operation of each module are local operations. Both partial and local operations act on the given module(s) but not necessarily on other modules. The set-theoretical union of all the partial and local operations gives a Brandt groupoid. The subset of these operations that act on the whole structure are global operations and they form the space group of the structure. We show that the groupoid analysis explains the structural relations between pairs of rods in each member of the series. We also show that the global operations obtained by analysing the groupoid of a given structure indeed result in the space group of that member. 